\title{
Analysis and Modeling of Echolocation Signals Emitted by Mediterranean Bottlenose Dolphins
}

\author{
Maria Greco and Fulvio Gini \\ Dipartimento di Ingegneria dell'Informazione, Elettronica, Informatica, Telecomunicazioni Università di Pisa, \\ via G. Caruso 16, 56122 Pisa, Italy
}

Received 21 January 2005; Revised 31 May 2005; Accepted 22 August 2005

Recommended for Publication by Jacques Verly

We analyzed the echolocation sounds emitted by Mediterranean bottlenose dolphins. We extracted the click trains by visual inspection of the data files recorded along the coast of the Tuscany with the collaboration of the CETUS Research Center. We modeled the extracted sonar clicks as Gaussian or exponential multicomponent signals, we estimated the characteristic parameters and compared the data with the reconstructed signals based on the estimates. Results about the estimation and the data fitting are largely shown in the paper.

Copyright () 2006 Hindawi Publishing Corporation. All rights reserved.

\section{INTRODUCTION}

Dolphins have a rich vocal repertoire that has been categorized into three classes:

(i) broadband, short-duration clicks, called sonar clicks, used in echolocation for orientation, perception, and navigation;

(ii) wideband pulsed sounds, called burst pulses, used in social contexts;

(iii) narrowband frequency-modulated whistles also used in social contexts.

This work is devoted to the analysis and modeling of echolocation signals emitted by the tursiops truncatus (bottlenose dolphin) living in the Tuscany Archipelago Park in both audio and ultrasonic bands.

Dolphins use a range of frequencies extending from 1 to $150 \mathrm{KHz}$. Communication signals (burst pulses and whistles) have a range of frequencies from 1 to $25 \mathrm{KHz}$. Generally, sonar signals have a range of frequencies from 25 to $150 \mathrm{KHz}$.

Dolphins can emit at the same time and independently sounds of various natures. Bottlenose dolphins have a remarkable range of hearing extending from less than $1 \mathrm{KHz}$ to more than $120 \mathrm{KHz}$ and a range of frequency-dependent sensitivity of nearly $100 \mathrm{~dB} \mu \mathrm{Pa}$. Dolphins have excellent frequency discrimination capability and are capable of determining changes in frequency as small as $0.2-0.4 \%$. This degree of discrimination is comparable to that observed in humans, but it is preserved across a much broader range of frequencies. The broad range of hearing and sensitivity and excellent frequency discrimination has likely evolved as part of the biological sonar system (echolocation) used by dolphins for exploitation of a visually limited marine environment. Dolphins respond to pure-tone signals in a similar manner as humans. Therefore, the spectral filtering property of the dolphin ear can be modeled by a bank of contiguous constant-Q filters, as for humans. Other hearing characteristics that are similar for dolphins and humans include frequency discrimination and sound localization capabilities in three-dimensional space.

Marine mammals do not use their mouths and throats to generate the sound-vocal chords rely on air. In dolphins, sound is produced below the nasal plug, and then focused by combination of reflection off the skull and passage through a lens mechanism formed by the melon, a mass of fatty tissue in the forehead [1]. The acoustic vibrations are then radiated from the bone of the rostrum into the blubber and sea water.

The acoustic field in the immediate vicinity of a dolphin head has no sharp null in the diagram of near-field and of beam. This is because short broadband pulses do not show effects of the constructive and destructive interference from multipath. The system of transmission of these pulses has the same irradiative characteristics of a directional antenna with $3 \mathrm{~dB}$ beampatterns of approximately $10^{\circ}$ on the vertical and horizontal planes. The beam is highly dependent on frequency, becoming narrower and narrower as the frequency 


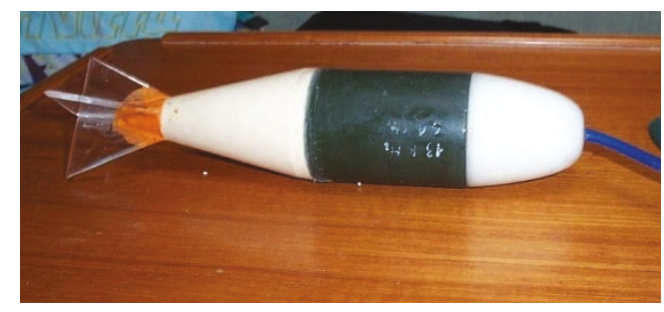

FIGURE 1: Hydrophone used in the data recording.

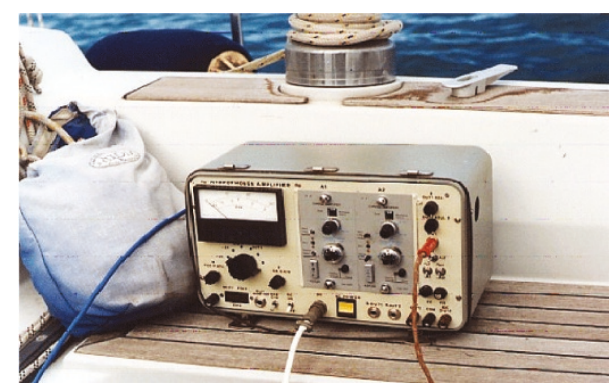

Figure 2: Amplifier used in the data recording.

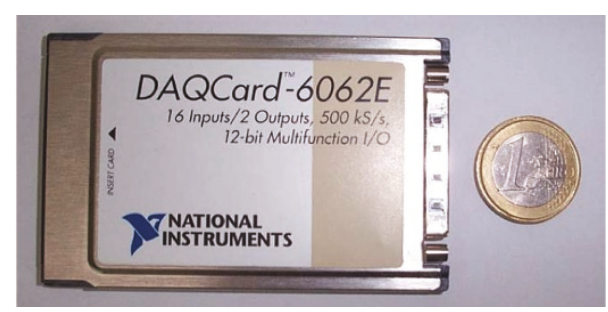

FIgURE 3: Digital card used in the data recording. increases. The directivity index of the transmitted beam pattern is approximately $26 \mathrm{~dB}$ in bottlenose dolphins [1].

Moreover, the emitted signal has different shapes according to the position of the animal with respect to the hydrophone. With an array of hydrophones, these different characteristics have been evidenced [1]. On the vertical plane (perpendicular to the head of the dolphin), the signal in the time domain became progressively distorted with respect to the signal on the major axis at $+5^{\circ}$; likewise, in the horizontal plane. The signals were not symmetrical about the beam axis, which is expected since the structure of the skull is not symmetrical about the midline of the animal [1].

\section{DATA ACQUISITION}

The chain of data acquisition and recording is composed by a hydrophone, a block of amplification, and a digital card on a laptop. In our recording, we first used a simple digital card with audio band $(0-16 \mathrm{KHz})$ and then we acquired by $\mathrm{Na}$ tional Instruments the digital card DAQCard-6062E, with a maximum sampling frequency of $5 \cdot 10^{5}$ samples per second.

The data acquisition has been made with the collaboration of the CETUS Research Center of Viareggio that since 1997 has monitered and has studied the cetaceans living in the Tuscany Archipelago.

\subsection{The hydrophone}

The interface between the acquisition system and the underwater world is represented by the hydrophone, an underwater microphone that converts a sound pressure in a proportional difference of tension. In Figure 1, we show the CETUS custom-built hydrophone used during our campaigns. Its body is a ceramic toroid sensible to the pressure. It works in the frequency range $(0 \mathrm{~Hz}-180 \mathrm{KHz})$ and it is almost omnidirectional. This characteristic can increase the possibility of recording sounds, but unfortunately, it can also prevent us from localizing their direction of arrival.

The hydrophone is dragged by the boat through a cable connected with the amplifier. This cable is $20 \mathrm{~m}$ long and it allows the hydrophone to stay generally $2 \mathrm{~m}$ below the surface, inside the thermoclyne. The cable is screened to avoid combinations with external signals, and shows a parasite power that is eliminated from the input stage of the amplifier. The cable vibrations also produce noise, at low frequencies, later eliminated by the amplifier. A small CETUS vessel was used to approach groups of dolphins in each locale.

\subsection{The amplifier}

The stage of amplification (see Figure 2) is composed by two charge amplifiers placed in cascade. The input impedance of the amplifier is about $10 \mathrm{M} \Omega$, and it has a bandpass behavior from $0 \mathrm{~Hz}$ up to $180 \mathrm{KHz}$. The amplifier also allows regulating manually the gain so we can always have the optimal level of signal during the recording. There is also an active highpass (HP) filter in the amplifier that removes the components of noise due to the boat engine, to the rinsing of the sea, to the vibrations of the cable carrying the hydrophone. The HP filter has a pole at $400 \mathrm{~Hz}$ with band of transition that decays $20 \mathrm{~dB} / \mathrm{dec}$. More details on the technical characteristics of the amplifier and of the hydrophone can be found in [2].

\subsection{Digital card}

During first recording days, we used a simple digital card with audio band $(0-16 \mathrm{KHz})$, then we acquired by National Instruments the digital card DAQCard-6062E (see Figure 3). This card allows recording even at ultrasonic band because its maximum sampling frequency is of $5 \cdot 10^{5}$ samples per second, then it is possible to catch signals until $250 \mathrm{KHz}$. In our files, dolphin echolocation signals were digitally sampled at a rate of $360 \mathrm{KHz}$, providing a Nyquist frequency for all recordings of $180 \mathrm{KHz}$, that is, the bandwidth of the hydrophone. Recordings were obtained from free-ranging bottlenose dolphins in the Mediterranean Sea, along the coast in front of Tuscany on 10 occasions. Audio band data were recorded 


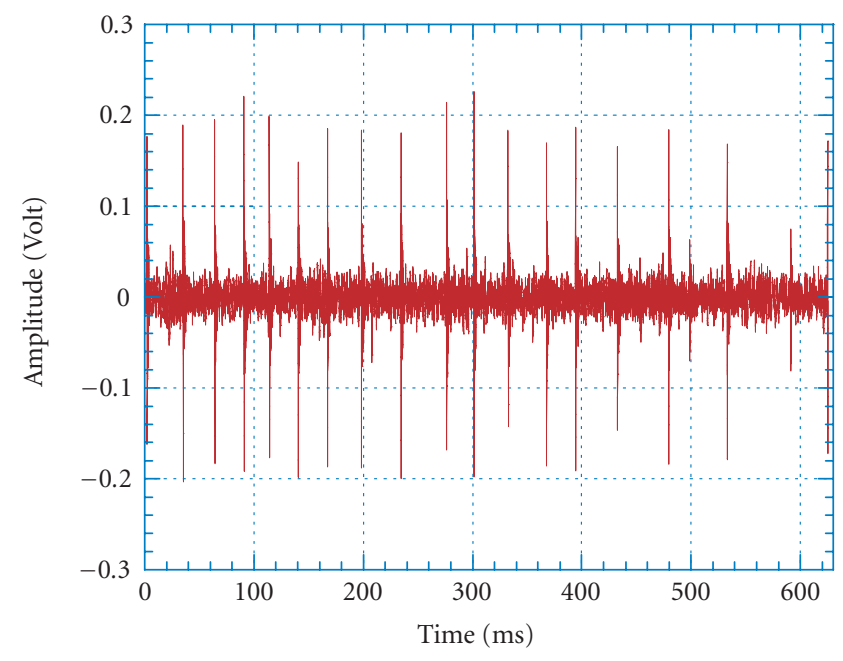

FIGURE 4: Sonar click train.

during various periods between June 2001 and September 2001. Ultrasonic signals were recorded during summer 2003.

\section{BIOSONAR MODEL}

The term sonar is the acronym for sound navigation and ranging and it was coined during the Second World War. It refers to the principle of detection and localization of objects in submarine environment, through emission of sonorous pulses and the processing of the echoes of return from the same objects. With the term echolocation is indicated the orientation ability using the transmission of ultrasonic pulses and the reception of the return echoes. The words sonar clicks, echolocation clicks, and biosonar are used to describe the activity of guideline, of navigation, and of localization of the animal that emits acoustic energy and analyzes the received echo. The first unequivocal demonstration of the use of the biosonar from dolphin dates back to 1960. Kenneth and Norris placed rubber suction cups over the eyes of a tursiop to eliminate its use of vision. The dolphin swam normally, emitting ultrasonic pulses and avoiding obstacles, including pipes suspended vertically to form a maze [3].

The dolphins use pulse trains as biosonar. A click train is plotted in Figure 4. The number of clicks and the temporal interval between successive clicks depend on several factors such as, for example, the distance from the target, the environmental conditions, and the expectation of the animal on the presence/absence of the prey. When the dolphin is in motion, the time that elapses between clicks often changes. A train of clicks can contain from just a few clicks to hundreds of clicks. If the pulses repeat rapidly, say every 5 milliseconds, we indifferently perceive them as a continuous tone [1]. Generally, the dolphin sends a click and waits for the return echo before sending the successive click. The time elapsing between the reception of the return echo and the emission of a new click (lag time) depends on the distance from the target.
From several studies $[1,4]$, it turns out that the mean lag time (LT) is 15 milliseconds with targets distant from $0.4 \mathrm{~m}$ to $4 \mathrm{~m}$, 2.5 milliseconds at less than $0.4 \mathrm{~m}$, and 20 milliseconds from $4 \mathrm{~m}$ to $40 \mathrm{~m}$. From several experiments, it is possible to assert that the dolphin can adapt the spectral content of the biosonar to the context in which they work in order to obtain the maximum efficiency [1] and the emitted pulses have duration that is different from a family to the other, in the range from ten to one hundred microseconds. The high resolution of biosonar and the ability to process the return echoes allows the dolphin to distinguish geometric figures, threedimensional objects, and to estimate the organic/inorganic composition of whichever object [1].

The biosonar signal has a peak-to-peak SPL (sound pressure level at a reference range of $1 \mathrm{~m}$ and a reference pressure of $1 \mu \mathrm{Pa}$ ) that varies between 120 and $230 \mathrm{~dB}$. The levels of SPL change considerably from family to family. The clicks of high level (greater than $210 \mathrm{~dB}$ ) introduce peaks of frequency at high frequency (hundreds of $\mathrm{KHz}$ ). Au et al. in fact postulated in $[1,4]$ that the high frequencies are by-product of producing high-intensity clicks. In other words, dolphins can only emit high-level clicks (greater than $210 \mathrm{~dB}$ ) if they use high frequencies. Dolphins maybe can emit high-frequency clicks at low amplitudes, but cannot produce low-frequency clicks at high amplitudes. Moreover, the dolphins can vary the amplitude of the biosonar in relation to the environmental conditions and to the distance of the target.

Frequency peaks are located between $5 \mathrm{KHz}$ and 150 $\mathrm{KHz}$. In open sea, the dolphins emit biosonar at high frequency with high level. In captivity, they produce echolocation clicks with peak frequencies an octave inferior and levels smaller than $15-30 \mathrm{~dB}$. This is because in open sea, there is much noise and the targets can be far, therefore a correct echolocation click can only happen through high frequency and high level. In captivity and in highly reverberant environment as the tanks of the aquarium, the close proximity of acoustic reverberant walls tends to discourage the animals from emitting high-intensity biosonars because too much energy would be reflected back to the dolphins [1].

In this paper, we describe methods for the analysis of recorded echolocation pulses and features extraction. The extracted information can be used by biologists to understand the ability of dolphins to perceive their environment and to perform difficult recognition and discrimination tasks, and also to relate the kind of emitted sounds to the behavior of these fascinating mammals.

The main focus is on the echolocation pulses recorded with the dolphins aligned to the hydrophone, that is, when the hydrophone is on the main axis of the dolphins. The study of measured data has been organized in four phases: classification, extraction, characterization, and estimation. In the first phase, all the recorded files have been classified by visual inspection. The time history and the timevarying spectrum of recorded data have been calculated to find the echolocation pulses. Subsequently, the interesting signals have been extracted from the files. In both audio and ultrasonic bands, we found visually mainly two kinds of pulses as shown in Figures 5(a)-5(b). The first pulse exhibits 


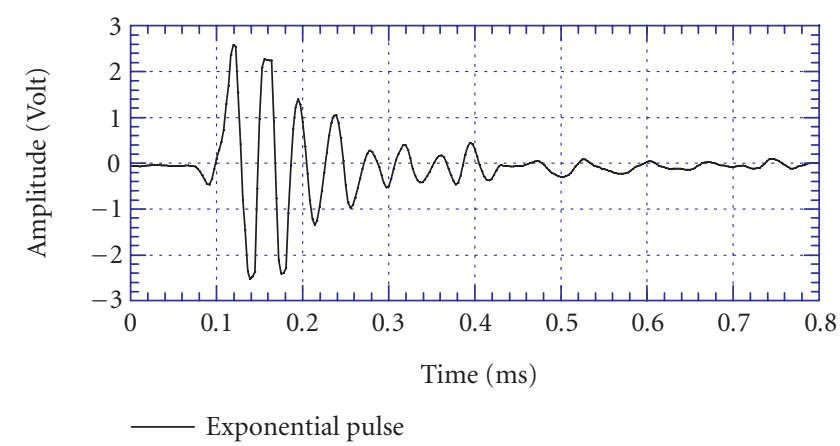

(a)

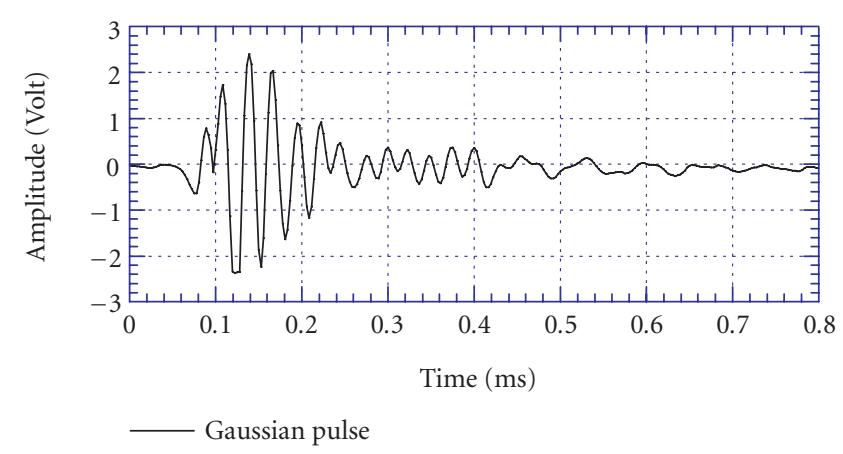

(b)

FIgUre 5: Exponential and Gaussian pulses extracted by data.

an exponential envelope, the second pulse a Gaussian envelope. For this study, we extracted 300 echolocation pulses from audio band data and more than 400 pulses in ultrasonic band. The analysis performed on the data for the sonar clicks is similar for both bands, and then we detail it for the ultrasonic band and resume the results for both frequency ranges.

\section{SIGNAL ESTIMATION}

\subsection{Exponential pulse}

For the sonar click of first kind, we adopted a dumped exponential multicomponent signal model, that is, we model the extracted signal $x(n)$ as

$$
x(n)=A_{0}+\sum_{k=1}^{K} A_{k} e^{-\alpha_{k} n} \cos \left(2 \pi f_{k} n+\vartheta_{k}\right),
$$

where $A_{0}$ is the mean value, $A_{k}, f_{k}$, and $\vartheta_{k}$ are amplitude, frequency, and initial phase of the $k$ th component, respectively, and $\alpha_{k}$ is the decay parameter of the exponential envelope. The signal (1) can be expressed in the more general form

$$
x(n)=A_{0}+\sum_{k=1}^{2 K} \beta_{k} e^{-\alpha_{k} n} e^{j 2 \pi f_{k} n},
$$

where $f_{k}=-f_{k+K}, \beta_{k}=\beta_{k+K}^{*}=A_{k} e^{j \vartheta_{k}} / 2$, and $\alpha_{k}=\alpha_{k+K}$.
To validate our model, we estimated the characteristic parameters using a least-square (LS) method. First of all, the mean value is estimated from the data as

$$
\widehat{A}_{0}=\frac{1}{N} \sum_{n=0}^{N-1} z(n)
$$

and subtracted from the data vector $z(n)=x(n)+w(n)$, where $w(n)$ is the additive noise, so obtaining the new data $y(n)=z(n)-\widehat{A}_{0}$. Then, the unknown parameter vector is $\boldsymbol{\theta}=\left[\beta_{1}, \ldots, \beta_{2 K}, \alpha_{1}, \ldots, \alpha_{2 K}, f_{1}, \ldots, f_{2 K}\right]=[\boldsymbol{\beta}, \boldsymbol{\alpha}, \mathbf{f}]$. Now define the cost function

$$
C(\mathbf{y} ; \boldsymbol{\theta})=\|\mathbf{y}-\mathbf{x}(\boldsymbol{\theta})\|^{2}=\frac{1}{N} \sum_{n=0}^{N-1}\left|y(n)-\sum_{k=1}^{2 K} \beta_{k} e^{-\alpha_{k} n} e^{j 2 \pi f_{k} n}\right|^{2},
$$

where $N$ is the number of samples describing a pulse and $\mathbf{y}$ is the data vector of length $N$. In audio band generally $N \simeq$ 100 , in ultrasonic band $N>400$. The nonlinear least-square (NLLS) estimator of $\boldsymbol{\theta}$ is

$$
\widehat{\boldsymbol{\theta}}=\arg \min _{\boldsymbol{\theta}} C(\mathbf{y} ; \boldsymbol{\theta}) .
$$

The estimators have the following expressions:

$$
\begin{gathered}
(\widehat{\mathbf{f}}, \widehat{\boldsymbol{\alpha}})=\arg \max _{\mathbf{f}, \alpha} \mathbf{y}^{H} \mathbf{A}\left(\mathbf{A}^{H} \mathbf{A}\right)^{-1} \mathbf{A}^{H} \mathbf{y}, \\
\widehat{\boldsymbol{\beta}}=\left(\mathbf{A}^{H} \mathbf{A}\right)^{-1} \mathbf{A}^{H} \mathbf{y},
\end{gathered}
$$

where $\mathbf{A}=\left[\mathbf{g}\left(\alpha_{1}\right) \odot \mathbf{p}\left(f_{1}\right) \cdots \mathbf{g}\left(\alpha_{2 K}\right) \odot \mathbf{p}\left(f_{2 K}\right)\right], \mathbf{a}\left(\alpha_{k}, f_{k}\right)=$ $\mathbf{g}\left(\alpha_{k}\right) \odot \mathbf{p}\left(f_{k}\right),[\mathbf{p}(f)]_{n}=e^{j 2 \pi f_{k} n},\left[\mathbf{g}\left(\alpha_{k}\right)\right]_{n}=g\left(n, \alpha_{k}\right)=e^{-\alpha_{k} n}$, and $\odot$ represents the element-by-element Hadamard product [5]. To reduce the computational complexity of the maximization in (6), we use a computationally efficient algorithm based on the RELAXation method $[6,7]$. It allows us to decouple the problem of jointly estimating the parameters of the signal components into a sequence of simpler problems, in which we estimate separately and iteratively the parameters of each component. RELAX first roughly estimates the parameters of the strongest component. It obtains the estimate $\hat{f}_{1}$ from the location of the highest peak of the periodogram [6] of the data $y$, then estimates the complex amplitude $\beta_{1}$ and the parameter $\alpha_{1}$ of the strongest component using the NLLS estimators for single component [2]. The contribution of the strongest component is subtracted from the data and the parameters of the new strongest second component are estimated. The procedure is iteratively repeated until "practical convergence" is achieved. This convergence is measured on the cost function $\operatorname{CF}\left(\left\{\hat{f}_{k}, \hat{\alpha}_{k}, \hat{\beta}_{k}\right\}_{k=1}^{P}\right)=$ $\sum_{n=0}^{N-1}\left|y(n)-\sum_{k=1}^{P} \hat{\beta}_{k} e^{-\hat{\alpha}_{k} n} e^{j 2 \pi \hat{f}_{k} n}\right|^{2}$, where $P=2$. Convergence is determined by checking the relative change of the cost function $\mathrm{CF}(\cdot)$ between the $j$ th and $(j+1)$ st iterations. In our numerical simulations, we terminated the iterations when the relative change is lower than $\varepsilon=10^{-4}$, as in [6]. When the convergence is achieved, the first two components are subtracted from the data and the parameters of the third one are estimated. The procedure is again iteratively repeated 
until convergence is achieved with the same cost function, where now $P=3$. The overall algorithm is repeated until the convergence for $P=2 K$ is achieved. Details on the relax are in $[2,6,7]$.

\subsection{Gaussian pulse}

For the sonar click of second kind, we adopted a dumped Gaussian multicomponent signal model, that is, we model the extracted signal $x(n)$ as

$$
x(n)=A_{0}+\sum_{k=1}^{K} A_{k} e^{-\alpha_{k}\left(n-n_{0 k}\right)^{2}} \cos \left(2 \pi f_{k} n+\vartheta_{k}\right),
$$

where $A_{0}$ is the mean value, $A_{k}, f_{k}$, and $\vartheta_{k}$ are amplitude, frequency, and initial phase of the $k$ th component, respectively. The model (8) is very similar to that proposed by Kamminga and Stuart in [8] where the authors use the Gabor functions. In that work, the number of components is fixed to two, the principal component and the reverberation; here $K$ can be greater than two to fit better the observed data. form

Again the signal (8) can be expressed in the more general

$$
x(n)=A_{0}+\sum_{k=1}^{2 K} \beta_{k} e^{-\alpha_{k}\left(n-n_{0 k}\right)^{2}} e^{j 2 \pi f_{k} n}
$$

where $f_{k}=-f_{k+K}, \beta_{k}=\beta_{k+K}^{*}=A_{k} e^{j \vartheta_{k}} / 2, \alpha_{k}=\alpha_{k+K}$, and $n_{0 k}=n_{0 k+K}$.

The difference between model (8) and (1) is the function characterizing the pulse envelope. In the model (1), it is an exponential function; in model (8), is a Gaussian function, that is, $\left[\mathbf{g}\left(\alpha_{k}, n_{0_{k}}\right)\right]_{n}=g\left(n, \alpha_{k}, n_{0_{k}}\right)=e^{-\alpha_{k}\left(n-n_{0 k}\right)^{2}}$. The exponential is characterized only by one parameter, the decay $\alpha$, the Gaussian function by two parameters, the scale parameter $\alpha$ and the mean value $n_{0}$. Therefore for the Gaussian model, there is one more parameter to estimate. In this case as well, we applied the NLLS estimation method and we implemented the relax algorithm to simplify the search for the maximum. The algorithm is very similar to that applied for the exponential shaped pulse.

The periodograms of an exponential and a Gaussian pulse are plotted in Figures 6(a)-6(b). For the analyzed exponential pulse, the main component is located around $25 \mathrm{KHz}$; for the Gaussian pulse, around $38 \mathrm{KHz}$.

\section{ESTIMATION RESULTS}

\subsection{Exponential pulse}

In our analysis, we set $K=2,3$, and 4 . We obtained a good fitting already for $K=2$. Here we show the results for $K=4$. In Figure 7, we show the scatterplot for the first two frequencies and exponential decays. It is evident that the first component (circles) is centered around 20$25 \mathrm{KHz}$ and spans almost the whole considered interval for the value of the exponential decay $\alpha_{1}$. The frequency of the second component is spread out on the interval $10-35 \mathrm{KHz}$. These results are confirmed by the histograms of frequencies

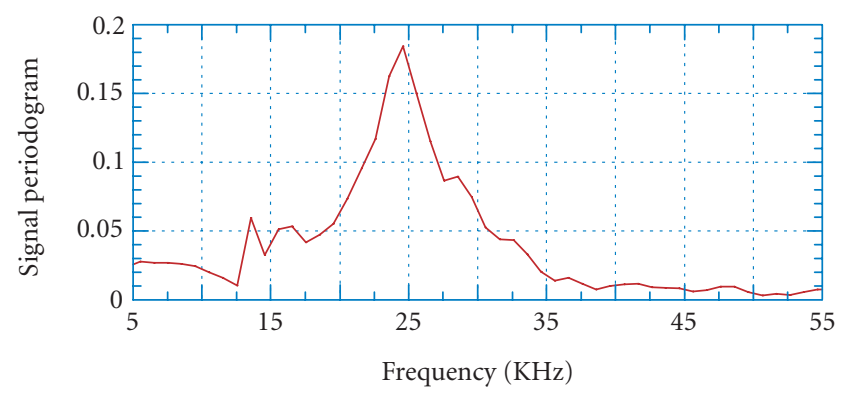

(a)

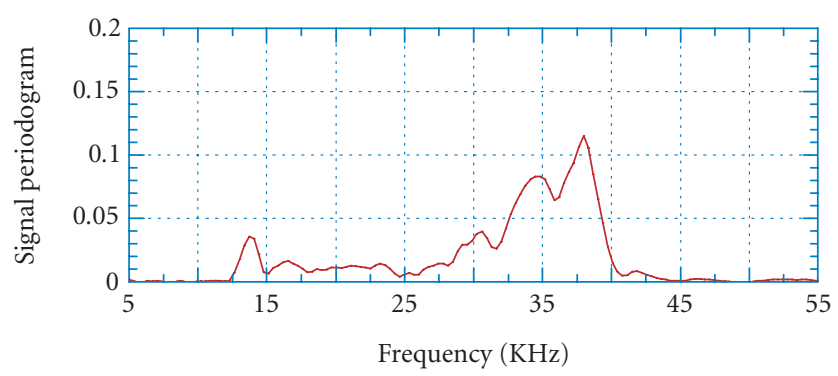

(b)

Figure 6: Signal periodogram for the exponential and Gaussian pulses in Figure 5(a) and 5(b), respectively.

and decays plotted in Figures 8 and 9. The first frequency (Figure 8(a)) has a Gaussian-like histogram with a mean value $\eta_{f_{1}}=23.59 \mathrm{KHz}$ and a standard deviation $\operatorname{std}\left\{f_{1}\right\}=$ $5.88 \mathrm{KHz}$. Conversely the second frequency (Figure $8(\mathrm{~b})$ ) is almost uniformly distributed in the range $(16 \mathrm{KHz}-32 \mathrm{KHz})$ with a mean value $\eta_{f_{2}}=24.28 \mathrm{KHz}$ and a standard deviation $\operatorname{std}\left\{f_{2}\right\}=8.32 \mathrm{KHz}$. The exponential decays exhibit Gaussian-like histograms with $\eta_{\alpha_{1}}=0.0177$, standard deviation $\operatorname{std}\left\{\alpha_{1}\right\}=0.0066, \eta_{\alpha_{2}}=0.0227$, and standard deviation $\operatorname{std}\left\{\alpha_{2}\right\}=0.010$, respectively (Figure 9). The third and fourth frequency components are almost uniformly distributed as well.

The mean and the standard deviation of each parameter have been respectively calculated as

$$
\begin{gathered}
\hat{\eta}_{\theta}=\frac{1}{N_{e}} \sum_{i=0}^{N_{e}-1} \theta_{i}, \\
\operatorname{std}\{\theta\}=\sqrt{\frac{1}{N_{e}} \sum_{i=0}^{N_{e}-1}\left(\theta_{i}-\hat{\eta}_{\theta}\right)^{2},}
\end{gathered}
$$

where $N_{e}$ is the number of estimates and $\theta_{i}$ the $i$ th estimate value of the generic parameter.

In Figure 10, we report the scatterplot of frequencies and amplitudes of the first two components. The amplitude is maximum when the frequency is comprised between 20 and $25 \mathrm{KHz}$. 


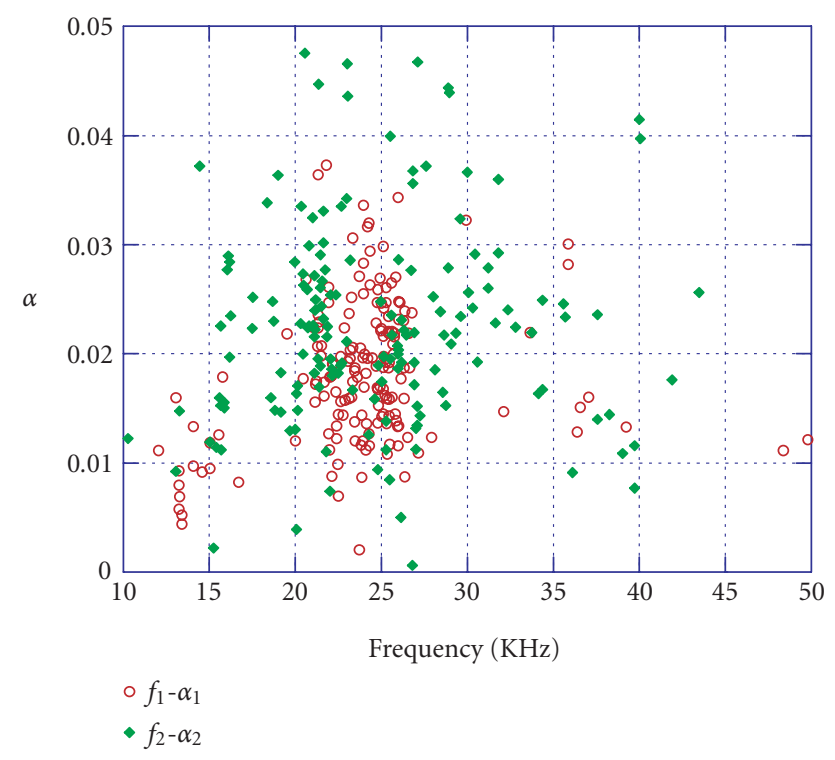

FIgURE 7: Scatterplot of frequency and exponential decay of first and second components, exponential model, $K=4$.

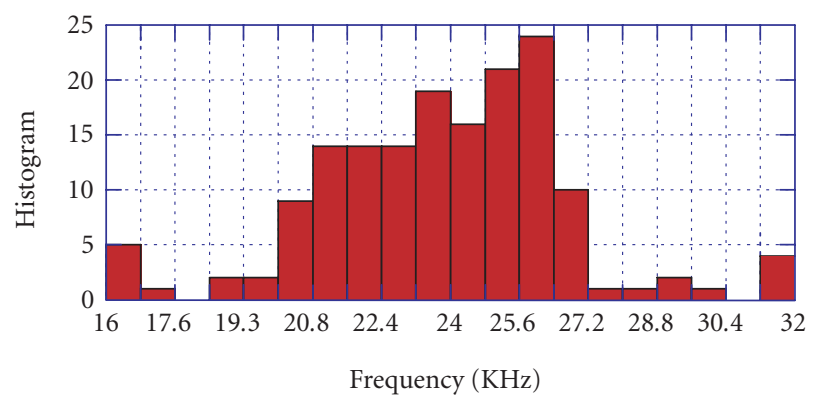

(a)

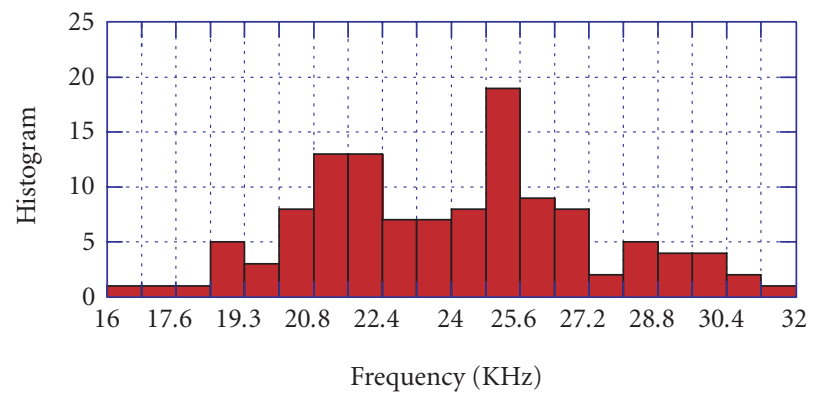

(b)

FIGURE 8: Histograms of the frequency of first and second components, exponential model, $K=4$.

From the results of Figures 8-10, we can observe that the component characterizing the exponential sonar clicks is the first one, the other components simply improve the fitting. This means that due to the almost uniform distribution of

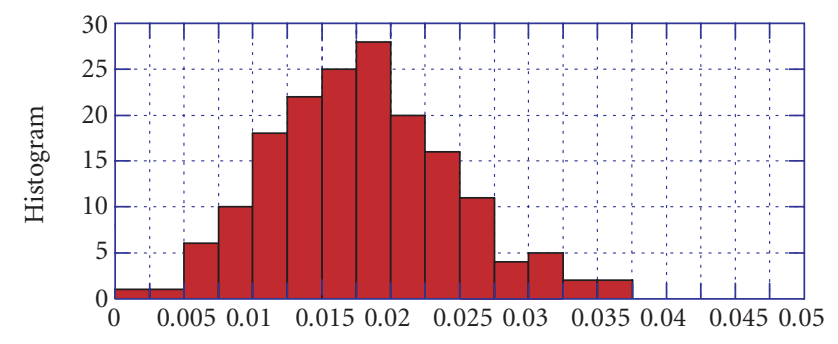

$\alpha_{1}$

(a)

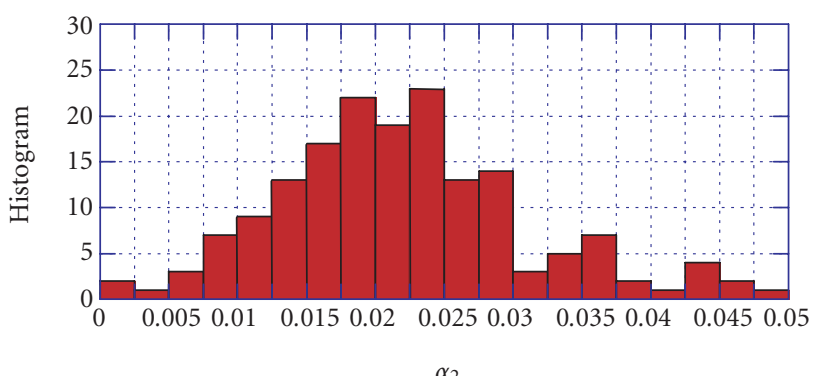

(b)

Figure 9: Histograms of the exponential decay parameter of first and second components, exponential model, $K=4$.

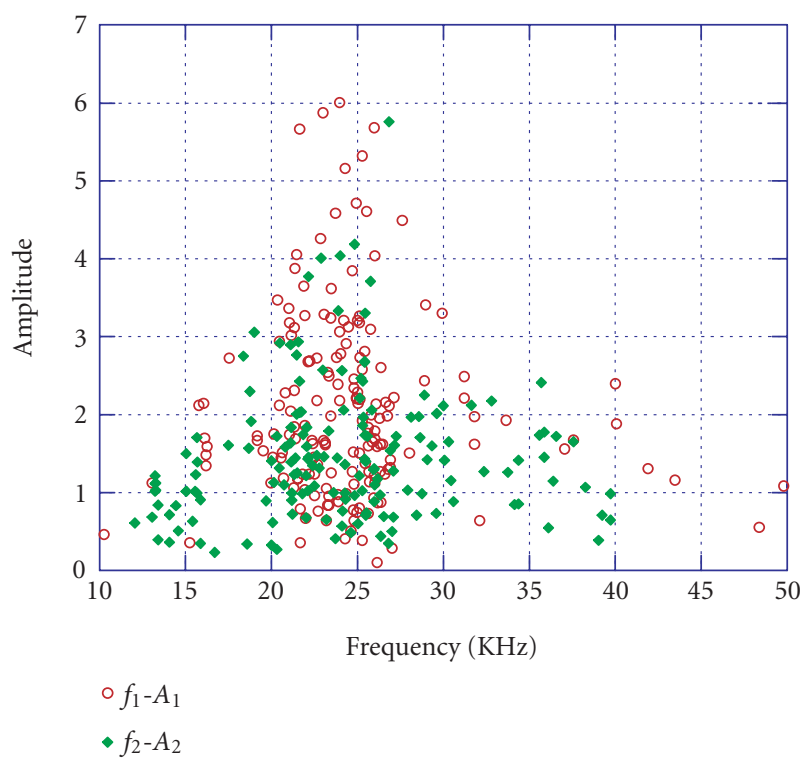

Figure 10: Scatterplot of frequency and amplitude of first and second components, exponential model, $K=4$.

the frequency of the second component, knowing this frequency does not help us to recognize the sonar pulse of one dolphin specie from another.

The mean values of the frequencies of all the four components are beyond the audio band. 


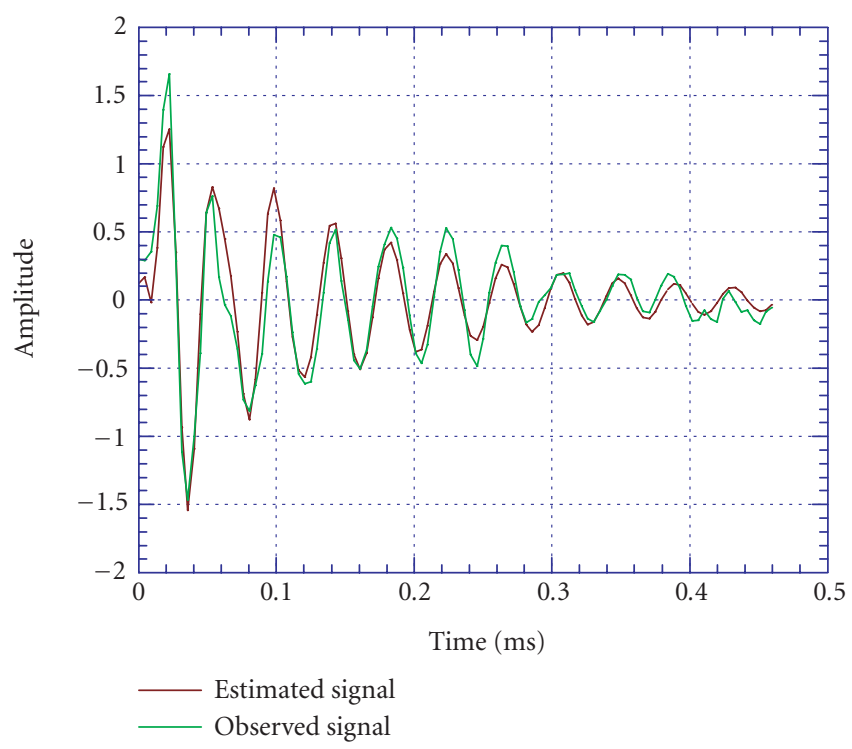

FIgURE 11: Fitting of an exponential pulse with the model (6) and $K=4$.

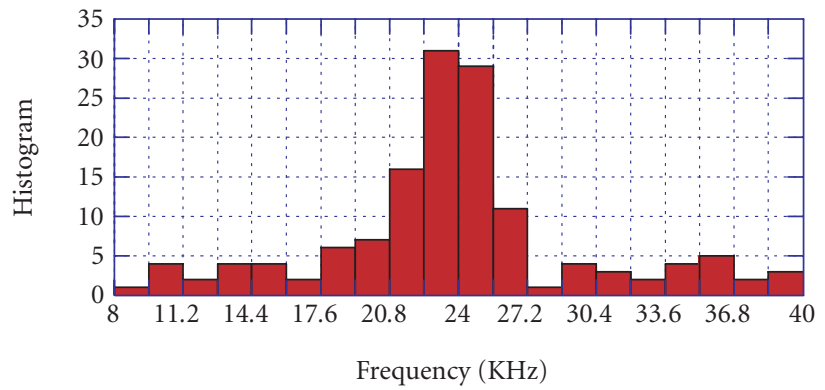

(a)

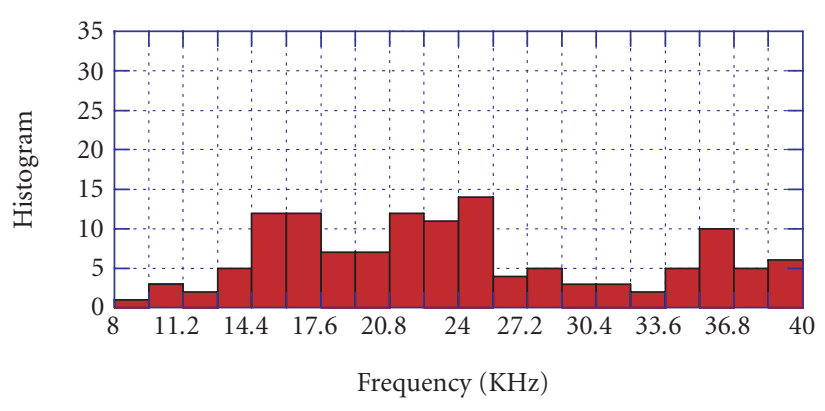

(b)

FIgURE 12: Histograms of the frequency of first and second components, Gaussian model, $K=4$.

In Figure 11, the observed and estimated signals are plotted for a sonar click for $K=4$. As apparent, the fitting of the exponential model is good.

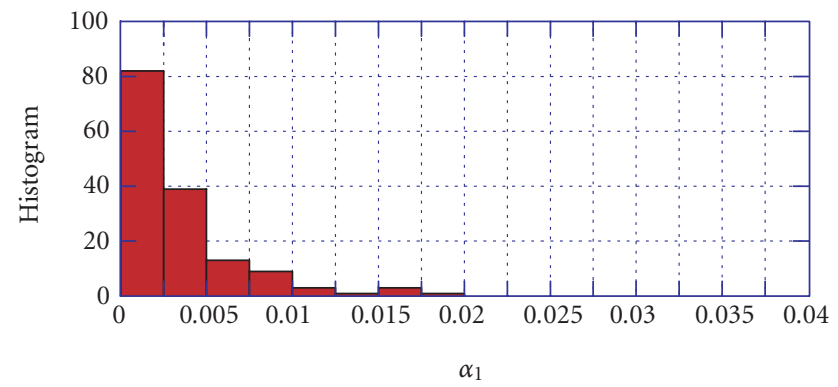

(a)

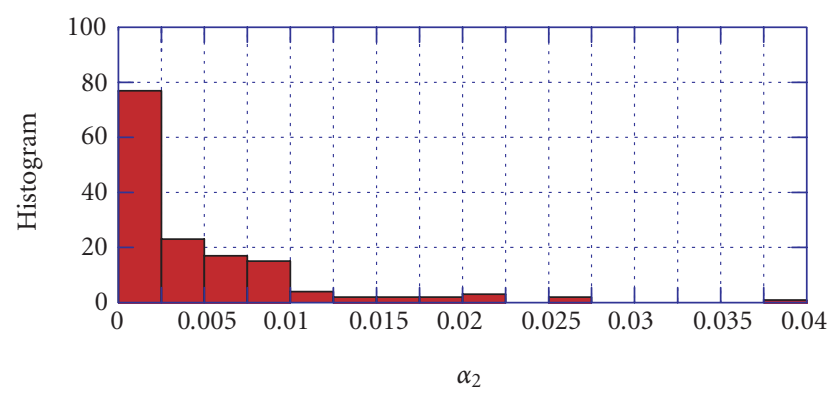

(b)

Figure 13: Histograms of the scale parameter of first and second components, Gaussian model, $K=4$.

\subsection{Gaussian pulse}

Similar analysis has been carried out on the clicks of the second kind and the results are reported in Figures 12, 13, 


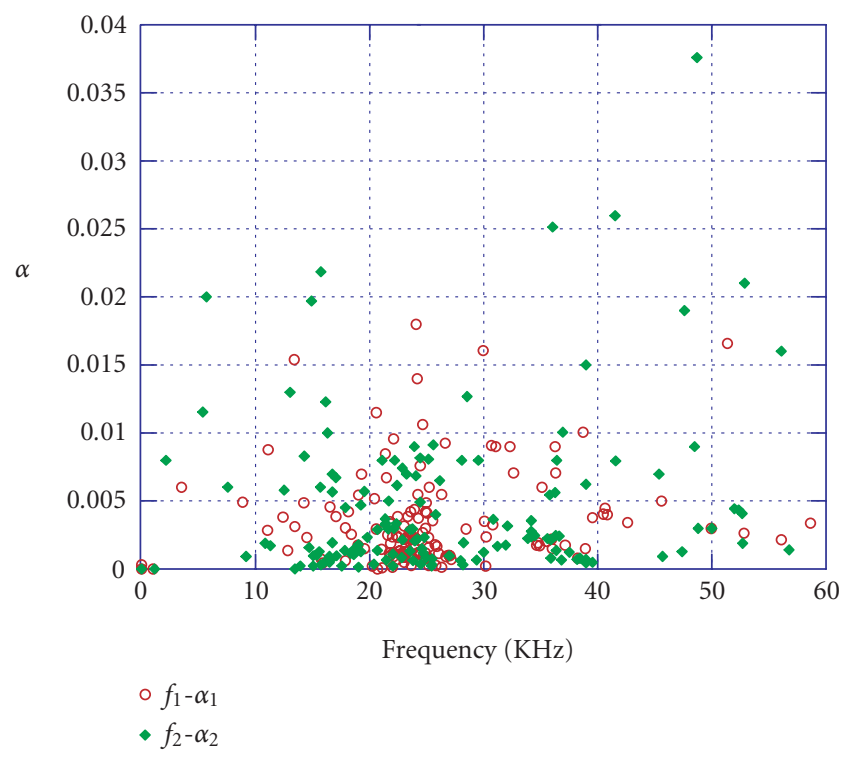

FIGURE 14: Scatterplot of frequency and scale parameter of first and second components, Gaussian model, $K=4$.

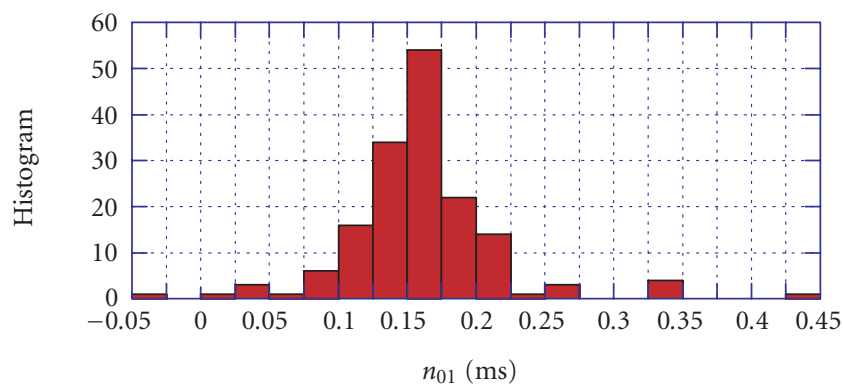

(a)

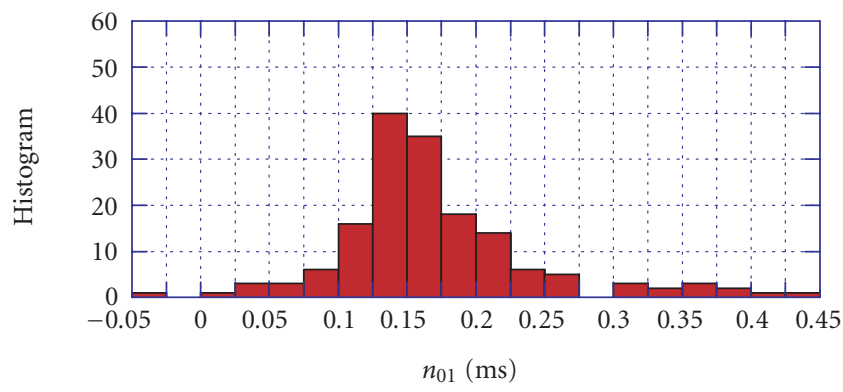

(b)

FIgURE 15: Histograms of time delay of first and second components, Gaussian model, $K=4$.

14,15 , and 16 for $K=4$. The frequency of the first component is concentrated in the interval $(21-27 \mathrm{KHz})$ with a mean value $\eta_{f_{1}}=25.83 \mathrm{KHz}$ and a normalized variance $\operatorname{var}\left\{f_{1}\right\}=0.186$, the frequency of the second component is almost uniformly distributed in $(14-40 \mathrm{KHz})$ with a mean value $\eta_{f_{1}}=27.21 \mathrm{KHz}$ and a normalized variance $\operatorname{var}\left\{f_{1}\right\}=$ 0.2723. (Figures 12 and 14). Both the scale factors exhibit a histogram with an exponential-like behavior in the range (00.02) as shown in Figures 13 and 14. Even the distributions of the time delays $n_{0_{1}}$ and $n_{0_{2}}$ of first and second components have a very similar Gaussian shape, but the mean value of the second component is greater than the first one, that is, the second Gaussian envelope is delayed with respect to the first one as shown in Figure 15; as a matter of fact, $E\left\{n_{0_{1}}\right\}=0.16$ 


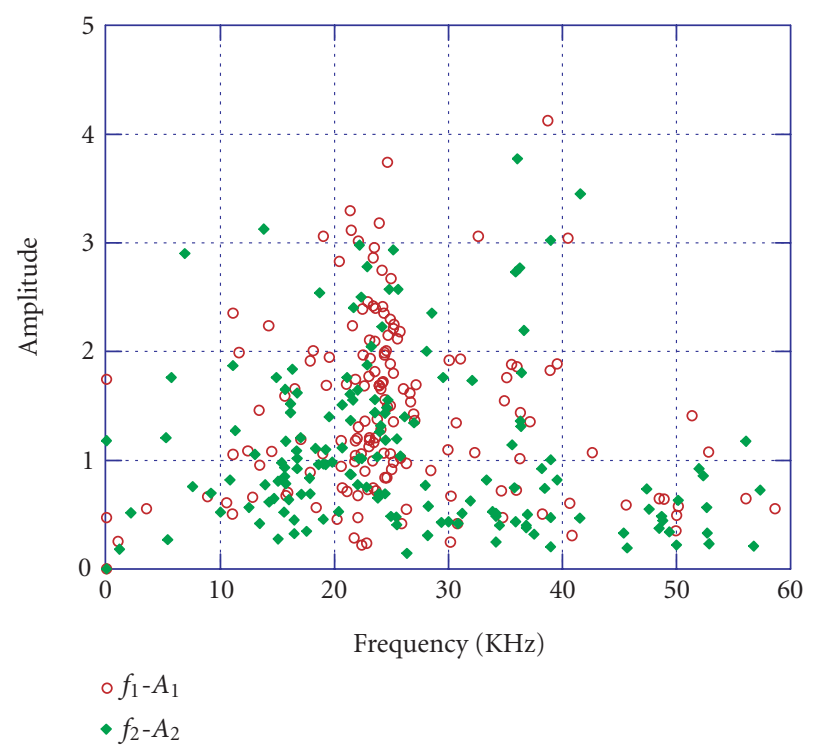

FIGURE 16: Scatterplot of frequency and amplitude of first and second components, Gaussian model, $K=4$.

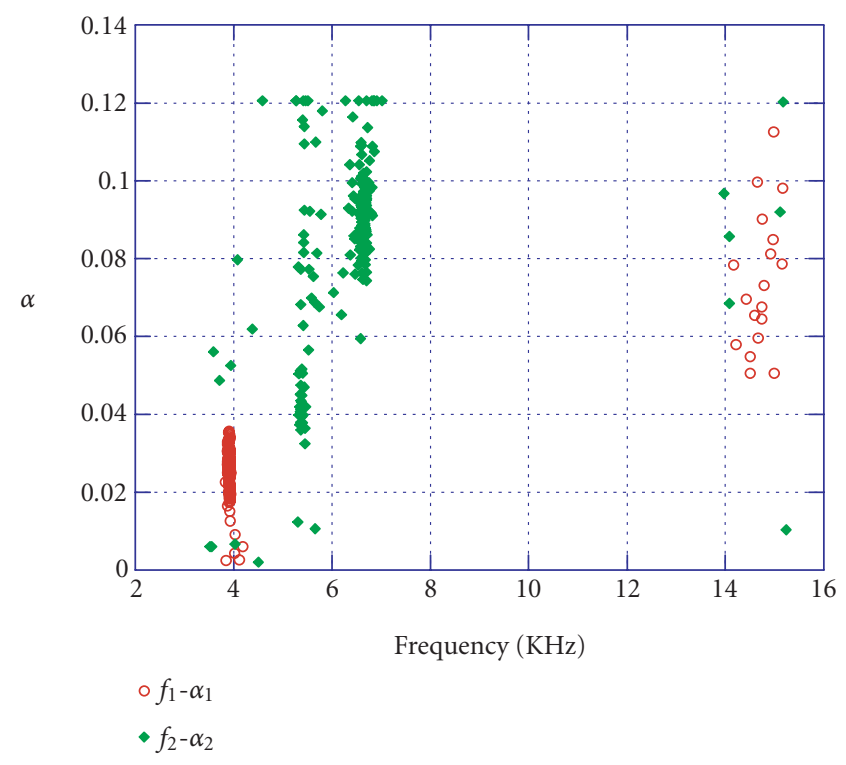

FIGURE 17: Scatterplot of frequency and exponential decay of first and second components, exponential model, $K=2$, audio band.

milliseconds and $E\left\{n_{0_{2}}\right\}=0.17$ milliseconds. The maximum amplitude corresponds to the components around $24 \mathrm{KHz}$ as shown in the scatterplot in Figure 16. Again, the dominant component is in the ultrasonic band.

We did not observe very high-frequency peaks in the sonar clicks emitted by the analyzed Mediterranean bottlenose dolphins as reported in literature for oceanic bottlenose dolphins [1]. This phenomenon could be mainly due to the difference in the environment. It is necessary to observe that those data referred to specimen living in the ocean and so in deep water and they use to move on long distances. To orientate, they use high-frequency and high-power biosonar. In fact, the dolphins cannot emit high-power signals at low frequency [1]. The cetaceans we are studying live in shallow waters, therefore they can use low-power signals and consequently low frequency.

\subsection{Audio band}

In analyzing the data recorded in the frequency range (0$180 \mathrm{KHz}$ ), we did not find even significant pulses at very low frequency. This fact can be easily understood by observing that usually in the dolphin emissions, higher frequency signals are characterized by higher power, then amplitude. The gain of the amplifier was manually changed during the recording in order to guarantee a good amplification and the absence of clipping even in presence of strong emissions. Doing so in the wide frequency range data, the low-power low-frequency pulses are completely covered by the electrical noise of the recording device.

Using the digital card of the laptop for audio signals, we recorded some files only in the audio band $(0-16 \mathrm{KHz})$. In these files, we extracted several exponential shaped sonar clicks. We analyzed these sonar click trains as in the ultrasonic band for $K=2$. The results are summarized in Figure 17 where the scatterplot of the estimated parameters $\left(\alpha_{1}, f_{1}\right)$ and $\left(\alpha_{2}, f_{2}\right)$ is reported. From this figure, it is well evident that the frequency of the first peak is almost constant around $3.8 \mathrm{KHz}$ for each pulse while its exponential decay $\left(\alpha_{1}\right)$ varies (lower vertical line) in the range $(0,0.038)$. The frequency of the second peak seems to have two more frequent values around $5.3 \mathrm{KHz}$ and $6.5 \mathrm{KH}$. The decay parameter varies sensibly in the range $(0,0.12)$ (the upper line). On the graph, there are some isolated points up to $14 \mathrm{KHz}$ due to a minority of very short pulses.

\section{CONCLUSIONS}

In this work, we analyze the sonar clicks emitted by Mediterranean bottlenose dolphins in both audio and ultrasonic bands. We found that most of the sonar clicks emitted when the dolphin is in front of the hydrophone can be modeled by and exponential or by Gaussian multicomponent signal. The parameters of these two models have been estimated. The components characterizing each pulse are generally the first or the first two most powerful and the fitting with the data seems to be very good in both audio and ultrasonic band. Actually, the meaning of the sonar clicks in the audio band signals is not clear. Maybe, as reported in [9], they can be "machinery noise," that is, noise produced by dolphins in emitting the ultrasonic pulses used for the echolocation. In ultrasonic band, the most powerful frequency component is located around $24 \mathrm{KHz}$, almost 4 octaves under the frequency peak measured for the oceanic bottlenose dolphins. This phenomenon can be mainly due to the differences in the oceanic and Mediterranean environments. 


\section{ACKNOWLEDGMENT}

This work has been partially funded by the European Project INTERREG IIIA.

\section{REFERENCES}

[1] W. W. L. Au, The Sonar of Dolphins, Springer, New York, NY, USA, 1993.

[2] M. Greco, F. Gini, L. Verrazzani, M. Mannucci, L. Alderani, and S. Nuti, Modeling and Feature Extraction of Audio Bio-Acoustic Signals Generated by Tyrrhenian Bottlenose Dolphins, Dipartimento di Ingegneria dell' Informazione, Università di Pisa, Pisa, Italy, October 2003.

[3] K. S. Norris, J. H. Prescott, P. V. Asa-Dorian, and P. Perkins, "An experimental demonstration of echolocation behavior in the porpoise, Tursiop truncatus, Montagu," Biological Bulletin, vol. 120, no. 2, pp. 163-176, 1961.

[4] W. W. L. Au, D. A. Carder, R. H. Penner, and B. L. Scronce, "Demonstration of adaptation in beluga whale echolocation signals," Journal of the Acoustical Society of America, vol. 77, no. 2, pp. 726-730, 1985.

[5] P. Stoica and R. Moses, Introduction to Spectral Analysis, Prentice-Hall, Upper Saddle River, NJ, USA, 1997.

[6] J. Li and P. Stoica, "Efficient mixed-spectrum estimation with applications to target feature extraction," IEEE Transactions on Signal Processing, vol. 44, no. 2, pp. 281-295, 1996.

[7] F. Gini, M. Greco, and A. Farina, "Multiple radar targets estimation by exploiting induced amplitude modulation induced by antenna scanning. part I: parameter estimation," IEEE Transactions on Aerospace and Electronic Systems, vol. 39, no. 4, pp. 1316-1332, 2003.

[8] C. Kamminga and A. B. C. Stuart, "Wave shape estimation of delphinid sonar signals, a parametric model approach," Acoustics Letters, vol. 19, no. 4, pp. 70-76, 1995.

[9] W. Zimmer, "Private communication," February 2004.

Maria Greco graduated in electronic engineering in 1993 and received the Ph.D. degree in telecommunication engineering in 1998, from University of Pisa, Italy. From December 1997 to May 1998, she joined the Georgia Tech Research Institute, Atlanta, USA, as a Visiting Research Scholar where she carried on research activity in the field of radar detection in non-Gaussian background. In 1993, she joined the Department

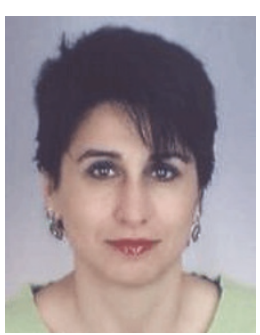
of "Ingegneria dell'Informazione" of the University of Pisa, where now she is an Assistant Professor since April 2001. She is IEEE Member since 1993 and she was a corecipient, with P. Lombardo, F. Gini, A. Farina, and B. Billingsley, of the 2001 IEEE Aerospace and Electronic Systems Society's Barry Carlton Award for Best Paper. Her general interests are in the areas of statistical signal processing, estimation and detection theory. In particular, her research interests include cyclostationarity signal analysis, bioacoustic signal analysis, clutter models, spectral analysis, coherent and incoherent detection in non-Gaussian clutter, and CFAR techniques. Dr. Greco has been a Session Chairman at international conferences and she is a coauthor of a tutorial entitled "Radar clutter modeling," presented at the International Radar Conference (May 2005, Arlington).
Fulvio Gini received the Doctor Engineer (cum laude) and the Ph.D. degrees in electronic engineering from the University of Pisa, Italy, in 1990 and 1995, respectively. In 1993 he joined the Department of "Ingegneria dell'Informazione" of the University of Pisa, where he is an Associate Professor since October 2000. He is an Associate Editor for the IEEE Transactions on Signal Processing and a Member of the EURASIP

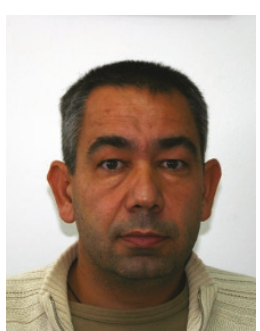
JASP Editorial Board. He was corecipient of the 2001 IEEE AES Society Barry Carlton Award for Best Paper. He was recipient of the 2003 IEE Achievement Award for outstanding contribution in signal processing and of the 2003 IEEE AES Society Nathanson Award to the Young Engineer of the Year. He is a Member of the SPTM and SAM Technical Committees of the IEEE SP Society. He is a Member of the Administrative Committee of the EURASIP Society and Award Chairman. He is Technical Co-chairman of the 2006 EUSIPCO Conference. His research interests include modeling and statistical analysis of recorded live sea and ground radar clutter data, non-Gaussian signal detection and estimation, parameter estimation and data extraction from multichannel interferometric SAR data, cyclostationary signal analysis, and estimation of nonstationary signals, with applications to radar signal processing. He authored or coauthored about 75 journal papers, about 70 conference papers, and two book chapters. 